\title{
On approximate homomorphisms of ternary semigroups
}

\author{
Krzysztof Ciepliński \\ AGH University of Science and Technology, Faculty of Applied Mathematics, Mickiewicza 30, 30-059 Krakow, Poland.
}

Communicated by J. Brzdek

\begin{abstract}
We prove the generalized Ulam stability of ternary homomorphisms from commutative ternary semigroups into $n$-Banach spaces as well as into complete non-Archimedean normed spaces. Ternary algebraic structures appear in various domains of theoretical and mathematical physics, and p-adic numbers, which are the most important examples of non-Archimedean fields, have gained the interest of physicists for their research in some problems coming from quantum physics, $p$-adic strings and superstrings. (C)2017 All rights reserved.
\end{abstract}

Keywords: Ulam stability, (commutative) ternary semigroup, ternary homomorphism, n-Banach space, (complete) non-Archimedean normed space, $\mathrm{p}$-adic numbers.

2010 MSC: 12J25, 17A40, 39B52, 39B82.

\section{Introduction}

The following natural question arises in many areas of scientific investigations: what errors we commit replacing functions satisfying some equations only approximately by exact solutions of these equations. Some efficient tools to evaluate these errors can be found in the theory of Ulam's stability.

Let us recall that we say that an equation is stable in a class of functions if any function from this class satisfying the equation approximately (in a sense), is near (in a way) to an exact solution of the equation. The problem of stability for homomorphisms of metric groups was formulated by Ulam in 1940, and its solution (for Banach spaces) was published a year later by Hyers.

In the last few decades, several stability problems of various (functional, differential, difference, integral) equations have been investigated by many mathematicians (see $[3,8]$ for the comprehensive accounts of the subject), but mainly in classical spaces. However, the notion of an approximate solution and the idea of nearness of two functions can be understood in various, nonstandard ways, depending on the needs and tools available in a particular situation. Such non-classical measures of a distance can be introduced for example by the notions of an $n$-norm and a non-Archimedean norm.

Let us recall (see for example $[1,2,17]$ ) that a pair $(G,[\cdot])$, where $G$ is a non-empty set and $[\cdot]: G^{3} \rightarrow G$ is a function (which is said to be a ternary operation), is called a ternary groupoid. Given a mapping $\oplus: \mathrm{G}^{2} \rightarrow \mathrm{G}$, we can define a ternary operation $[\cdot]$ on $\mathrm{G}$ by

$$
[x y z]:=(x \oplus y) \oplus z, \quad x, y, z \in G .
$$

Email address: cieplin@agh.edu.pl (Krzysztof Ciepliński)

doi:10.22436/jnsa.010.08.03 
Then we say that the operation $[\cdot]$ is derived from $\oplus$. In this note, every linear space will be treated as a ternary groupoid with an operation derived from vector addition.

A ternary groupoid is said to be commutative if

$$
\left[x_{1} x_{2} x_{3}\right]=\left[x_{\sigma(1)} x_{\sigma(2)} x_{\sigma(3)}\right], \quad x_{1}, x_{2}, x_{3} \in G, \quad \sigma \in S_{3},
$$

where $S_{3}$ denotes the set of all permutations of the set $\{1,2,3\}$.

We say that a ternary groupoid $(G,[\cdot])$ is a ternary semigroup if the operation $[\cdot]$ is associative, i.e., if

$$
[[x y z] u v]=[x[y z u] v]=[x y[z u v]], \quad x, y, z, u, v \in G .
$$

It is obvious that every linear space (see the remark above) is a ternary semigroup.

Such ternary algebraic structures appear in various domains of theoretical and mathematical physics (for example "Nambu mechanics" the 4-dimensional Minkowskian space-time, and the algebra of "nonions", which was introduced by Sylvester as a ternary analog of Hamilton's quaternions; see [1, 9, 13] for details).

Let $\left(G_{1},[\cdot]_{1}\right)$ and $\left(G_{2},[\cdot]_{2}\right)$ be ternary groupoids. A mapping $f: G_{1} \rightarrow G_{2}$ is called a ternary homomorphism if

$$
f\left([x y z]_{1}\right)=[f(x) f(y) f(z)]_{2}, \quad x, y, z \in G_{1} .
$$

In 2006, Amyari and Moslehian (see [1]) proved the generalized Ulam stability of ternary homomorphisms from commutative ternary semigroups into Banach spaces. In this note, we generalize their result to $n$-Banach spaces as well as we extend it to complete non-Archimedean normed spaces (for other outcomes on the stability of some mappings defined on ternary algebraic structures see for example [15, 16]).

Throughout this paper $\mathbb{N}$ stands for the set of all positive integers, $\mathbb{N}_{0}:=\mathbb{N} \cup\{0\}$ and $\mathbb{R}_{+}:=[0, \infty)$. We will also write $x^{3}$ instead of $[x x x]$.

\section{Stability of ternary homomorphisms with values in n-Banach spaces}

In 1989, Misiak (see [12]) defined n-normed spaces and studied their properties. The concept of an n-normed space is a generalization of the notions of a classical normed space and of a 2-normed space introduced by Gähler. More information on these spaces and on some problems investigated in them can be found for instance in $[5,6,7,11,19]$.

\subsection{Preliminaries}

Now, we recall some basic definitions and facts concerning n-normed spaces (for more details and some examples, we refer the reader to $[6,7,12,19])$.

Let $n \in \mathbb{N}$, $X$ be an at least $n$-dimensional real linear space and $\|\cdot, \cdots, \cdot\|: X^{n} \rightarrow \mathbb{R}_{+}$be a function satisfying the following conditions:

(N1) $\left\|x_{1}, \cdots, x_{n}\right\|=0$ if and only if $x_{1}, \cdots, x_{n}$ are linearly dependent;

(N2) $\left\|x_{1}, \cdots, x_{n}\right\|$ is invariant under permutation;

(N3) $\left\|\alpha x_{1}, \cdots, x_{n}\right\|=|\alpha|\left\|x_{1}, \cdots, x_{n}\right\|$;

(N4) $\left\|x+y, x_{2}, \cdots, x_{n}\right\| \leqslant\left\|x, x_{2}, \cdots, x_{n}\right\|+\left\|y, x_{2}, \cdots, x_{n}\right\|$

for any $\alpha \in \mathbb{R}$ and $x, y, x_{1}, \cdots, x_{n} \in X$. Then the function $\|\cdot, \cdots, \cdot\|$ is called an $n$-norm on $X$, and a pair $(X,\|\cdot, \cdots, \cdot\|)$ is said to be an $n$-normed space.

A sequence $\left(y_{k}\right)_{k \in \mathbb{N}}$ of elements of an $n$-normed space $(X,\|\cdot, \cdots, \cdot\|)$ is called a Cauchy sequence if

$$
\lim _{k, l \rightarrow \infty}\left\|y_{k}-y_{l}, x_{2}, \cdots, x_{n}\right\|=0, \quad x_{2}, \cdots, x_{n} \in X,
$$

whereas $\left(y_{k}\right)_{k \in \mathbb{N}}$ is said to be convergent if there exists a $y \in X$ (called the limit of this sequence and 
denoted by $\lim _{k \rightarrow \infty} y_{k}$ ) with

$$
\lim _{k \rightarrow \infty}\left\|y_{k}-y, x_{2}, \cdots, x_{n}\right\|=0, \quad x_{2}, \cdots, x_{n} \in X .
$$

An n-normed space in which every Cauchy sequence is convergent is called an n-Banach space.

Let us also mention that in n-normed spaces every convergent sequence has exactly one limit and the standard properties of the limit of a sum and a scalar product are valid.

In what follows, we will use the following lemma from [19].

Lemma 2.1. Let $(\mathrm{X},\|\cdot, \cdots, \cdot\|)$ be an $\mathrm{n}$-normed space. Then:

(i)

$$
\left\|x_{1}, \cdots, x_{i}, \cdots, x_{j}, \cdots, x_{n}\right\|=\left\|x_{1}, \cdots, x_{i}, \cdots, x_{j}+\alpha x_{i}, \cdots, x_{n}\right\|
$$

for any $x_{1}, \cdots, x_{n} \in X, \alpha \in \mathbb{R}$ and $i, j \in\{1, \cdots, n\}$ with $i \neq j$;

(ii)

$$
\left\|x, y_{2}, \cdots, y_{n}\right\|-\left\|y, y_{2}, \cdots, y_{n}\right\| \mid \leqslant\left\|x-y, y_{2}, \cdots, y_{n}\right\|
$$

for any $x, y, y_{2}, \cdots, y_{n} \in X$;

(iii) if $x \in X$ and

then $x=0$;

$$
\left\|x, y_{2}, \cdots, y_{n}\right\|=0, \quad y_{2}, \cdots, y_{n} \in X
$$

(iv) if $\left(x_{k}\right)_{k \in \mathbb{N}}$ is a convergent sequence of elements of $X$, then

$$
\lim _{k \rightarrow \infty}\left\|x_{k}, y_{2}, \cdots, y_{n}\right\|=\left\|\lim _{k \rightarrow \infty} x_{k}, y_{2}, \cdots, y_{n}\right\|, \quad y_{2}, \cdots, y_{n} \in X
$$

\subsection{Main results}

In this subsection, we show the generalized Ulam stability of ternary homomorphisms from commutative ternary semigroups into $n$-Banach spaces. Some results on the stability of functional equations in $n$-Banach spaces can be found for example in $[4,19]$ (see also the references given there).

Theorem 2.2. Let $\mathrm{G}$ be a ternary semigroup, $\mathrm{n} \in \mathbb{N}, \mathrm{X}$ be an $\mathrm{n}$-Banach space, and $\varphi: \mathrm{G}^{3} \rightarrow \mathbb{R}_{+}$be a function with

$$
\widehat{\varphi}(x, y, z):=\frac{1}{3} \sum_{k=0}^{\infty} 3^{-k} \varphi\left(x^{3^{k}}, y^{3^{k}}, z^{3^{k}}\right)<\infty, \quad(x, y, z) \in G^{3} .
$$

If $\mathrm{f}: \mathrm{G} \rightarrow \mathrm{X}$ is a mapping such that

$$
\left\|f([x y z])-(f(x)+f(y)+f(z)), x_{2}, \cdots, x_{n}\right\| \leqslant \varphi(x, y, z)
$$

for $(x, y, z) \in G^{3}$ and $x_{2}, \cdots, x_{n} \in X$, then there exists a unique function $T: G \rightarrow X$ satisfying

$$
\left\|f(x)-T(x), x_{2}, \cdots, x_{n}\right\| \leqslant \widehat{\varphi}(x, x, x), \quad x \in G, x_{2}, \cdots, x_{n} \in X,
$$

and

$$
\mathrm{T}\left(\mathrm{x}^{3}\right)=3 \mathrm{~T}(\mathrm{x}), \quad \mathrm{x} \in \mathrm{G} .
$$

If, moreover, the semigroup $\mathrm{G}$ is commutative, then $\mathrm{T}$ is a ternary homomorphism.

Proof. Putting $z=y=x$ in (2.2) we get

$$
\left\|f\left(x^{3}\right)-3 f(x), x_{2}, \cdots, x_{n}\right\| \leqslant \varphi(x, x, x), \quad x \in G, x_{2}, \cdots, x_{n} \in X,
$$

whence, by induction,

$$
\left\|3^{-k} f\left(x^{3^{k}}\right)-f(x), x_{2}, \cdots, x_{n}\right\| \leqslant \frac{1}{3} \sum_{j=0}^{k-1} 3^{-j} \varphi\left(x^{3^{j}}, x^{3^{j}}, x^{3^{j}}\right)
$$

for $x \in G, x_{2}, \cdots, x_{n} \in X$ and $k \in \mathbb{N}$. Consequently, 


$$
\left\|3^{-k} f\left(x^{3^{k}}\right)-3^{-l} f\left(x^{3^{l}}\right), x_{2}, \cdots, x_{n}\right\| \leqslant \frac{1}{3} \sum_{j=l}^{k-1} 3^{-j} \varphi\left(x^{3^{j}}, x^{3^{j}}, x^{3^{j}}\right)
$$

for $x \in G, x_{2}, \cdots, x_{n} \in X$ and $k, l \in \mathbb{N}$ with $l<k$. Therefore from (2.1) it follows that for every $x \in G$, $\left(3^{-j} f\left(x^{3^{j}}\right)\right)_{j \in \mathbb{N}}$ is a Cauchy sequence. Since $X$ is an $n$-Banach space, this sequence is convergent and we can put

$$
\mathrm{T}(\mathrm{x}):=\lim _{j \rightarrow \infty} 3^{-j} f\left(x^{3^{j}}\right), \quad x \in \mathrm{G} .
$$

It is clear that then (2.4) holds. Moreover, letting $k \rightarrow \infty$ in (2.5) and using (2.1) and Lemma 2.1 we get (2.3).

Next, assume that $T^{\prime}: G \rightarrow X$ is another mapping satisfying conditions (2.3) and (2.4), and fix $x \in$ $\mathrm{G}, \mathrm{x}_{2}, \cdots, \mathrm{x}_{\mathrm{n}} \in \mathrm{X}$ and $\mathrm{k} \in \mathbb{N}$. Then

$$
\begin{aligned}
\left\|T(x)-T^{\prime}(x), x_{2}, \cdots, x_{n}\right\| & =\left\|3^{-k} T\left(x^{3^{k}}\right)-3^{-k} T^{\prime}\left(x^{3^{k}}\right), x_{2}, \cdots, x_{n}\right\| \\
& \leqslant 3^{-k}\left(\left\|T\left(x^{3^{k}}\right)-f\left(x^{3^{k}}\right), x_{2}, \cdots, x_{n}\right\|+\left\|f\left(x^{3^{k}}\right)-T^{\prime}\left(x^{3^{k}}\right), x_{2}, \cdots, x_{n}\right\|\right) \\
& \leqslant 2 \cdot 3^{-k} \widehat{\varphi}\left(x^{3^{k}}, x^{3^{k}}, x^{3^{k}}\right)=\frac{2}{3} \cdot \sum_{j=k}^{\infty} 3^{-j} \varphi\left(x^{3^{j}}, x^{3^{j}}, x^{3^{j}}\right),
\end{aligned}
$$

whence letting $k \rightarrow \infty$ we obtain

$$
\left\|T(x)-T^{\prime}(x), x_{2}, \cdots, x_{n}\right\|=0,
$$

and Lemma 2.1 now shows that $\mathrm{T}=\mathrm{T}^{\prime}$.

Let us finally assume that the semigroup $\mathrm{G}$ is commutative. Then, by (2.2),

$$
\begin{aligned}
\| 3^{-j} f\left([x y z]^{3^{j}}\right) & -3^{-j}\left(f\left(x^{3^{j}}\right)+f\left(y^{3^{j}}\right)+f\left(z^{3^{j}}\right)\right), x_{2}, \cdots, x_{n} \| \\
& \leqslant 3^{-j} \varphi\left(x^{3^{j}}, y^{3^{j}}, z^{3^{j}}\right), \quad j \in \mathbb{N}, \quad(x, y, z) \in G^{3}, x_{2}, \cdots, \quad x_{n} \in X,
\end{aligned}
$$

whence letting $j \rightarrow \infty$ and using (2.1), Lemma 2.1 and (2.6) we get

$$
\left\|\mathrm{T}([x y z])-(\mathrm{T}(\mathrm{x})+\mathrm{T}(\mathrm{y})+\mathrm{T}(z)), x_{2}, \cdots, x_{\mathrm{n}}\right\|=0
$$

for $(x, y, z) \in G^{3}, x_{2}, \cdots, x_{n} \in X$, which in view of Lemma 2.1 proves that $T$ is a ternary homomorphism.

Theorem 2.2 with $\varphi \equiv \varepsilon>0$ yields immediately the following result on classical Ulam stability of ternary homomorphisms under consideration.

Corollary 2.3. Let $\mathrm{G}$ be a ternary semigroup, $\mathrm{n} \in \mathbb{N}, \mathrm{X}$ be an $\mathrm{n}$-Banach space and $\varepsilon>0$. If $\mathrm{f}: \mathrm{G} \rightarrow \mathrm{X}$ is a mapping such that

$$
\left\|f([x y z])-(f(x)+f(y)+f(z)), x_{2}, \cdots, x_{n}\right\| \leqslant \varepsilon
$$

for $(x, y, z) \in \mathrm{G}^{3}$ and $x_{2}, \cdots, x_{n} \in X$, then there exists a unique function $T: G \rightarrow X$ satisfying condition (2.4) and

$$
\left\|f(x)-T(x), x_{2}, \cdots, x_{n}\right\| \leqslant \frac{\varepsilon}{2}, \quad x \in G, x_{2}, \cdots, x_{n} \in X .
$$

If, moreover, the semigroup $\mathrm{G}$ is commutative, then $\mathrm{T}$ is a ternary homomorphism.

\section{Stability of ternary homomorphisms with values in complete non-Archimedean normed spaces}

The first work on the Ulam stability of functional equations in complete non-Archimedean normed spaces (some particular cases were considered earlier; see [3] for details) was [14]. After it a lot of papers (see, for instance, $[3,18]$ and the references given there) on the stability of different equations in such spaces have been published. 


\subsection{Preliminaries}

Let us recall (see for example [10,14]) some basic definitions and facts concerning non-Archimedean normed spaces.

By a non-Archimedean field we mean a field $\mathbb{K}$ equipped with a function (called a valuation) $|\cdot|: \mathbb{K} \rightarrow$ $\mathbb{R}_{+}$such that

$$
\begin{gathered}
|r|=0, \quad \text { if and only if } \quad r=0, \\
|r s|=|r||s|, \quad r, s \in \mathbb{K},
\end{gathered}
$$

and

$$
|r+s| \leqslant \max \{|r|,|s|\}, \quad r, s \in \mathbb{K} .
$$

In any non-Archimedean field we have $|1|=|-1|=1$ and $|\mathfrak{n}| \leqslant 1$ for $n \in \mathbb{N}_{0}$.

In any field $\mathbb{K}$ the function $|\cdot|: \mathbb{K} \rightarrow \mathbb{R}_{+}$given by

$$
|x|:= \begin{cases}0, & x=0 \\ 1, & x \neq 0\end{cases}
$$

is a valuation which is called trivial, but the most important examples of non-Archimedean fields are p-adic numbers which have gained the interest of physicists for their research in some problems coming from quantum physics, $p$-adic strings and superstrings.

Let $X$ be a linear space over a field $\mathbb{K}$ with a non-Archimedean non-trivial valuation $|\cdot|$. A function $\|\cdot\|: X \rightarrow \mathbb{R}_{+}$is said to be a non-Archimedean norm if it satisfies the following conditions:

$$
\begin{gathered}
\|x\|=0, \quad \text { if and only if } \quad x=0, \\
\|r x\|=|r|\|x\|, \quad r \in \mathbb{K}, \quad x \in X,
\end{gathered}
$$

and

$$
\|x+y\| \leqslant \max \{\|x\|,\|y\|\}, \quad x, y \in X .
$$

Then $(X,\|\cdot\|)$ is called a non-Archimedean normed space. In any such a space the function $d: X \times X \rightarrow \mathbb{R}_{+}$ given by

$$
d(x, y)=\|x-y\|, \quad x, y \in X
$$

is a metric on $X$.

Recall also that a sequence $\left(x_{n}\right)_{n \in \mathbb{N}}$ of elements of a non-Archimedean normed space is Cauchy if and only if the sequence $\left(x_{n+1}-x_{n}\right)_{n \in \mathbb{N}}$ converges to zero. Moreover, the addition, scalar multiplication and non-Archimedean norm are continuous mappings.

\subsection{Main result}

In this section, we show the generalized Ulam stability of ternary homomorphisms from commutative ternary semigroups into complete non-Archimedean normed spaces.

Theorem 3.1. Let $\mathrm{G}$ be a ternary semigroup, $\mathrm{X}$ be a complete non-Archimedean normed space over a nonArchimedean field of the characteristic different from $3, \varphi: \mathrm{G}^{3} \rightarrow \mathbb{R}_{+}$be a function such that

$$
\lim _{k \rightarrow \infty}|3|^{-k} \varphi\left(x^{3^{k}}, y^{3^{k}}, z^{3^{k}}\right)=0, \quad(x, y, z) \in G^{3},
$$

and for each $x \in \mathrm{G}$ the limit

$$
\lim _{k \rightarrow \infty} \max \left\{|3|^{-j} \varphi\left(x^{3^{j}}, x^{3^{j}}, x^{3^{j}}\right): 0 \leqslant j<k\right\},
$$

denoted by $\widehat{\varphi}(\mathrm{x})$, exists. If $\mathrm{f}: \mathrm{G} \rightarrow \mathrm{X}$ is a mapping such that

$$
\|f([x y z])-(f(x)+f(y)+f(z))\| \leqslant \varphi(x, y, z), \quad(x, y, z) \in G^{3},
$$


then there exists a function $\mathrm{T}: \mathrm{G} \rightarrow \mathrm{X}$ satisfying condition (2.4) and

$$
\|f(x)-T(x)\| \leqslant \frac{1}{|3|} \widehat{\varphi}(x), \quad x \in G .
$$

If, moreover, the semigroup $\mathrm{G}$ is commutative, then $\mathrm{T}$ is a ternary homomorphism.

Proof. Putting $z=y=x$ in (3.2) we get

$$
\left\|f\left(x^{3}\right)-3 f(x)\right\| \leqslant \varphi(x, x, x), \quad x \in G,
$$

whence

$$
\left\|3^{-k} f\left(x^{3^{k}}\right)-3^{-k+1} f\left(x^{3^{k-1}}\right)\right\| \leqslant|3|^{-k} \varphi\left(x^{3^{k-1}}, x^{3^{k-1}}, x^{3^{k-1}}\right)
$$

for $x \in G, k \in \mathbb{N}$. Therefore from (3.1) it follows that for every $x \in G,\left(3^{-j} f\left(x^{3^{j}}\right)\right)_{j \in \mathbb{N}}$ is a Cauchy sequence. Since $X$ is a complete non-Archimedean normed space, this sequence is convergent and we can define $T: G \rightarrow X$ by (2.6). It is clear that then (2.4) holds.

Next, by induction, we show that

$$
\left\|3^{-k} f\left(x^{3^{k}}\right)-f(x)\right\| \leqslant \frac{1}{|3|} \max \left\{|3|^{-j} \varphi\left(x^{3^{j}}, x^{3^{j}}, x^{3^{j}}\right): 0 \leqslant j<k\right\}
$$

for $x \in G, k \in \mathbb{N}$, whence letting $k \rightarrow \infty$ and using the definitions of $\widehat{\varphi}$ and $T$ we get (3.3).

Let us finally assume that the semigroup $\mathrm{G}$ is commutative. Then, by (3.2),

$$
\left\|3^{-j} f\left([x y z]^{3^{j}}\right)-3^{-j}\left(f\left(x^{3^{j}}\right)+f\left(y^{3^{j}}\right)+f\left(z^{3^{j}}\right)\right)\right\| \leqslant|3|^{-j} \varphi\left(x^{3^{j}}, y^{3^{j}}, z^{3^{j}}\right), \quad j \in \mathbb{N}, \quad(x, y, z) \in G^{3},
$$

whence letting $j \rightarrow \infty$ and using (3.1) we conclude that $\mathrm{T}$ is a ternary homomorphism.

\section{References}

[1] M. Amyari, M. S. Moslehian, Approximate homomorphisms of ternary semigroups, Lett. Math. Phys., 77 (2006), 1-9. 1

[2] N. Bazunova, A. Borowiec, R. Kerner, Universal differential calculus on ternary algebras, Lett. Math. Phys., 67 (2004), 195-206. 1

[3] N. Brillouët-Belluot, J. Brzdęk, K. Ciepliński, On some recent developments in Ulam's type stability, Abstr. Appl. Anal., 2012 (2012), 41 pages. 1, 3

[4] H.-Y. Chu, A. Kim, J. Park, On the Hyers-Ulam stabilities of functional equations on n-Banach spaces, Math. Nachr., 289 (2016), 1177-1188. 2.2

[5] H. Dutta, On some n-normed linear space valued difference sequences, J. Franklin Inst., 348 (2011), 2876-2883. 2

[6] G. P. Gehér, On n-norm preservers and the Aleksandrov conservative n-distance problem, ArXiv, 2015 (2015), 9 pages. 2, 2.1

[7] H. Gunawan, M. Mashadi, On n-normed spaces, Int. J. Math. Math. Sci., 27 (2001), 631-639. 2, 2.1

[8] S.-M. Jung, Hyers-Ulam-Rassias stability of functional equations in nonlinear analysis, Springer Science and Business Media, New York, (2011). 1

[9] R. Kerner, Ternary and non-associative structures, Int. J. Geom. Methods Mod. Phys., 5 (2008), 1265-1294. 1

[10] A. Khrennikov, Non-Archimedean analysis: quantum paradoxes, dynamical systems and biological models, Kluwer Academic Publishers, Dordrecht, (1997). 3.1

[11] Y. Ma, The Aleksandrov-Benz-Rassias problem on linear n-normed spaces, Monatsh. Math., 180 (2016), 305-316. 2

[12] A. Misiak, n-inner product spaces, Math. Nachr., 140 (1989), 299-319. 2, 2.1

[13] M. S. Moslehian, Ternary derivations, stability and physical aspects, Acta Appl. Math., 100 (2008), 187-199. 1

[14] M. S. Moslehian, T. M. Rassias, Stability of functional equations in non-Archimedean spaces, Appl. Anal. Discrete Math., 1 (2007), 325-334. 3, 3.1

[15] C. Park, M. E. Gordji, Comment on "Approximate ternary Jordan derivations on Banach ternary algebras" [Bavand Savadkouhi et al. J. Math. Phys. 50, 042303 (2009)], J. Math. Phys., 2010 (2010), 7 pages. 1

[16] J. M. Rassias, H.-M. Kim, Approximate homomorphisms and derivations between C*-ternary algebras, J. Math. Phys., 2008 (2008), 10 pages. 1

[17] M. L. Santiago, S. Sri Bala, Ternary semigroups, Semigroup Forum, 81 (2010), 380-388. 1

[18] T. Z. Xu, Stability of multi-Jensen mappings in non-Archimedean normed spaces, J. Math. Phys., 2012 (2012), 9 pages. 3

[19] T. Z. Xu, J. M. Rassias, On the Hyers-Ulam stability of a general mixed additive and cubic functional equation in n-Banach spaces, Abstr. Appl. Anal., 2012 (2012), 23 pages. 2, 2.1, 2.2 